\title{
Status of India-based Neutrino Observatory Project
}

\author{
Naba K Mondal ${ }^{1}$ \\ Tata Institute of Fundamental Research \\ Homi Bhabha Road, Mumbai 400005, India \\ E-mail: nkmetifr.res.in
}

\begin{abstract}
We discuss the current status of the India-based Neutrino Observatory (INO) proposal to setup an underground laboratory in India and to construct a 50 kton magnetized iron calorimeter (ICAL) detector for observing atmospheric neutrino interactions. We give a brief description of the detector and discuss its physics motivations. The charge identification capability of ICAL would make it complementary to large water Cerenkov and other detectors world wide. The status of the design of the magnet, the construction of the prototype detector and the development of glass resistive plate chambers (RPC) are presented.
\end{abstract}

35th International Conference of High Energy Physics (ICHEP2010)

Paris, France

July 22-28, 2010

1 Speaker 


\section{Introduction}

India-based Neutrino Observatory (INO) is a newly proposed underground facility in the southern part of India. It is proposed to construct an underground laboratory with a large cavern of dimensions $132 \mathrm{~m}$ X $26 \mathrm{~m}$ X $20 \mathrm{~m}$ to house a 50 kton magnetized iron tracking calorimeter detector (ICAL) to study atmospheric neutrinos. In addition a smaller cavern of size $55 \mathrm{~m} \times 12.5 \mathrm{~m} \times 8.6 \mathrm{~m}$ will also be constructed to host other smaller experiments. There will be at least $1.2 \mathrm{~km}$ rock overburden in all directions. All the details of INO can be found in the INO project report [1].

\section{ICAL Detector}

The proposed INO-ICAL detector will have a modular structure of total lateral size $48 \mathrm{~m} \times 16 \mathrm{~m}$ and will consist of a stack of 150 horizontal layers of $\sim 5.6 \mathrm{~cm}$ thick magnetized iron plates interleaved with $4 \mathrm{~cm}$ gaps to house the active detector layers. The ICAL detector will be subdivided into three modules of size $16 \mathrm{~m} \times 16 \mathrm{~m}$. This modular structure will allow early operation with the completed modules while constructing others. The iron structure for this detector will be self supporting with the layer above resting on the layer immediately below using iron spacers located every $2 m$ along the $\mathrm{X}$ direction. This will create $2 m$ wide roads along the Y-direction for the insertion of active detector elements. The iron plates will be magnetized with a field strength of $\sim 1.4$ tesla to determine the charge of the muon produced by neutrino interaction inside the detector, so that the $\nu_{\mu}$ and $\bar{\nu}_{\mu}$ induced events could be studied separately. The magnetic field will also help to measure the momentum of the final state particles, specially the muons produced in the neutrino interactions inside the ICAL detector. The active detector elements are resistive plate chambers (RPCs), made up of a pair of $3 \mathrm{~mm}$ thick glass plates of area $2 m \times 2 m$, separated by $2 \mathrm{~mm}$ spacers. They are operated at a high voltage of about $9.9 \mathrm{KV}$ in avalanche mode. A high energy charged particle, passing through RPC, leaves a signal with an efficiency of 90-95\%. The read out of the RPCs will be performed by external orthogonal X \& Y pickup strips of $3 \mathrm{~cm}$ wide allowing determination of the $\mathrm{x}$ and $\mathrm{y}$ coordinates of the passage of the charged particles through the RPC. The RPC location will provide the $\mathrm{Z}$ coordinate. The time resolution of $\sim 1 \mathrm{nsec}$ will enable one to distinguish upward going particles from downward going particles. Hence from the hit pattern observed in the RPCs, the energy and direction of the charged particles produced in the neutrino interaction can be reconstructed. Fig. 1 shows the over all layout of INO ICAL detector.

\section{Physics Motivation}

The main physics goals of INO are: 
(i) Reconfirmation with greater statistical significance the first oscillation dip in $L / E$ of the atmospheric neutrinos, and measure $\left|\Delta_{31}\right| \approx\left|\Delta m^{2}{ }_{\text {atm }}\right|$ and $\sin ^{2} 2 \theta_{23}$ precisely.

(ii) Determine the sign of $\Delta m^{2}{ }_{31}$ and hence the neutrino mass hierarchy using matter effect.

(iii) Measure the deviation of $\theta_{23}$ from maximality, and resolve the octant ambiguity.

(iv) Distinguish $\quad v_{\mu} \leftrightarrow v_{\tau}$ from $v_{\mu} \leftrightarrow v_{s}$ oscillation from muonless events.

(v) Search for CPT violation.

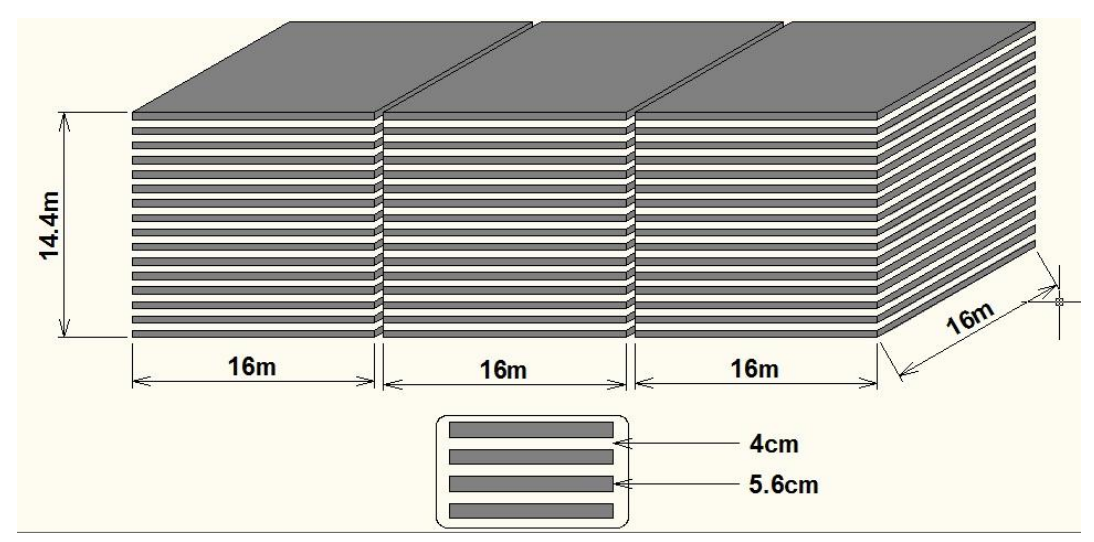

Figure 1: Schematic view of the 50 kton iron calorimeter (ICAL)

\section{Status of the Project}

An extensive R \& D program for the development of INO detector is currently ongoing. As mentioned earlier, the active detector elements will be $2 m \times 2 m$ glass RPCs with $X$ and $Y$ position resolution of $\sim 2 \mathrm{~cm}$ and a time resolution $\sigma_{t} \sim 1 \mathrm{nsec}$. A vertical stack of $121 \mathrm{~m}^{2}$ glass RPCs has been setup and being operated continuously to track cosmic ray muons. The collaboration is also working on the development of a small prototype ICAL detector including the magnet with 12 layers of iron plates of 5 $\mathrm{cm}$ thick and $121 \mathrm{~m}^{2}$ glass RPCs placed in between the iron plates. It will be operational soon. A gas mixing and circulation system has been developed in collaboration with a local vendor. A collaborative project to delelop a close loop gas mixing and gas recirculation system including a condenser-purifier is also being persued. Similarly vendors for the conducting coating on the outer surface of the glass plates of the RPCs for applying high voltage, polycarbonate spacers and buttons and gas inlet/outlet nozzles have been developed. The detailed project report (DPR) for the site and associated infrastructure for the underground and over ground laboratories has been prepared. One additional DPR for the mechanical structure and assembly of the ICAL magnet and the ancillary mechanical facilities is also ready. We expect the construction work for excavation to start within next six months.

\section{References}

[1] INO Project Report, INO/2006/01 